\title{
VIBRATIONAL ENERGY FLOWS IN BEAM NETWORKS WITH COMPLIANT AND DISSIPATIVE JOINTS
}

\author{
M. BESHARA \\ Department of Engineering Science, University of Oxford, Parks Road, Oxford OX13PJ, \\ England \\ AND \\ A. J. KeANE \\ Department of Mechanical Engineering, University of Southampton, Highfield, \\ Southampton SO17 1BJ, England
}

(Received 6 September 1996, and in final form 10 December 1996)

\begin{abstract}
Many structures and machines are made up from components (rods, beams and plates) which are joined together by welds, bolts or rivets. The mechanical behaviour of such built-up systems is greatly affected by the properties of their joints which are usually compliant and dissipative.

In this work, a general solution for the vibrational energy flows through a plane network of beams is sought, based on the receptance approach. The joints between any two elements are assumed to act at discrete points and are modelled by three sets of springs and dashpots, thus being compliant and non-conservative in all the three degrees of freedom relevant to this case. The beams are assumed to be slender and elastic, and the deflections at the joint are assumed to be small, so that conventional linear beam theory may be used in the analysis. The aim of this study is to give greater insight into the problem of non-conservative coupling, which has not been extensively discussed in the literature. Interest is focused on the effect of damping in the joints on the magnitudes of energy flows between, and energy levels in, each beam. Variations in the energy flows through a compliant joint between two beams with changes in their coupling angle are also discussed. Numerical examples which illustrate these various ideas are presented.

(C) 1997 Academic Press Limited
\end{abstract}

\section{INTRODUCTION}

The joint properties of built-up structures greatly affect their mechanical behaviour. In many studies, however, the dynamics of assembled structures have been based on the assumption of rigid joints. Among those who have adopted this approach are: Langley [1], who adopted the dynamic stiffness method to find the mean power flow and stored energy for a general framework with rigid joints; Shankar and Keane [2], who evaluated the vibrational energies of a framework of beams using a receptance approach; Cuschieri [3], who used the mobility approach to predict the structural energy flow through the joint between two plates when rigidly coupled together; Goyder and White [4], who predicted the vibrational energy flow from machines into built-up structures using the frequency response characteristics of an equivalent infinite structure; Cremer et al. [5], who studied the transmission of waves through rigid joints of different configurations to find wave transmission coefficients; and Horner and White [6], who used the same approach to find the wave transmission coefficients of vibrational power transmitted through rigid bends in built-up structures. 
It is clear, however, that the joints in real structures are often compliant, and this may greatly"affect the behaviour of an assembled structure and the transmission of vibrational power through its components. Davies [7, 8] studied the energy flows between two beams coupled together where the joint had compliance in rotation, modelling this by a rotational spring. Keane [9] studied a network of rods in an arbitrary configuration coupled together by linear springs and derived expressions for the energy flow through the joints, based on a receptance approach. Even so, these studies did not deal with dissipative joints. Moreover, it has been shown in previous work [10] that the damping encountered at the joints in real structures often dissipates more energy than the internal damping of the components, and it has been long recognized that this characteristic may be employed successfully in vibration control problems.

To overcome these limitations, a number of researchers have used spring and dashpot models to represent dissipative joints and have found that a linear spring-viscous dashpot combination is adequate for modelling such joints in many cases; see, for example, Gaul [10] or Yoshimura and Okushima [11]. Interest has also been focused on the study of the energy flow through dissipative joints and the way in which it is affected by the presence of coupling damping; see Fahy and Yao [12] and Sun et al. [13]. These studies also discussed the effects of coupling damping on the main energy balance equations of Statistical Energy Analysis (SEA) [14] and suggested modifications to the subsystem internal loss factors to take into account the presence of coupling damping. Recently, Leung and Pinnington $[15,16]$ used the wave approach to analyze wave propagation through a right-angled joint between two beams, where the joint was modelled by three sets of springs and dashpots, which allowed compliance in the three degrees of freedom. Their results show the effect of the presence of damping at the joint on the transmission of energy, represented by the wave transmission coefficients. However, in that work it was assumed that the beams were infinite in length, and solutions were not given for a general joint configuration where the angle between the connected beams is arbitrary. Rook and Singh [17] outlined a framework for the analysis of energy flow through compliant and dissipative joints, based on the mobility and modal approaches. In their formula, the structure is decomposed into three substructures where the joints were considered non-conservative and compliant, again modelled by springs and viscous dampers. However, their formulae are deduced only for finite-degree-of-freedom systems and are only strictly accurate when using the entire modal basis, which is practically impossible for any real structure, leading to modal truncation errors (although such errors may be slight if sufficient number of modes are used for any given problem). The case of multiple, non-conservatively coupled systems has also been studied recently by Beshara and Keane [18], using a receptance approach. This method gives the energy flows in terms of the Green functions of the uncoupled systems and the characteristics of the joints, which are assumed to act at discrete points. However, the study was limited to the case of multiple rods, which can only support longitudinal waves, and therefore a solution was not given for the general case of a network of beams which can support multiple wave types.

In this paper that work is extended and expressions are presented for the energy flows in a plane network of beams with compliant and dissipative joints, based on a receptance approach, where the expressions are again written in terms of the receptance functions of the uncoupled beams and the properties of the joints. Thus, the effect of the joint properties on the energy flows can be efficiently investigated. Here the power dissipated at the joint between two beams is studied and the conditions under which it is maximized are highlighted. The effects of changes in the angle between the beams on the magnitudes of the energy flows are also examined, and it is noted that the variation of energy flow with 
angle shows different patterns depending on the flexural and longitudinal modal densities of the coupled beams.

It may additionally be noted that studies dealing with compliant joints also belong to the field of "flexible-link mechanism" analysis. This field is still relatively little explored, but is gaining increasing attention from industry, because such mechanisms have some advantages over those with non-compliant joints [19], and this is therefore an important and developing branch of mechanics. Howell and Midha [19] have developed a general method for the design of compliant mechanisms and give a flowchart for the design process.

\section{THEORY}

In this work, a plane network of beams with compliant and dissipative joints is analyzed and general expressions for the energy flows, energy dissipations at the joints and the energy levels of the beams are recovered, based on a receptance approach. It is assumed that the joints between any two elements are massless and of small dimensions compared with the beam elements, which they connect at discrete points. A compliant and dissipative joint is modelled here by three sets of springs and dashpots, as in previous studies $[15,16]$. The beams are assumed to be slender and elastic, and deflections at the joint are assumed to be small, so that conventional linear beam theory may be used in the analysis. The joint properties are also assumed to be invariant with frequency. The basic approach adopted follows that already described by Beshara and Keane [18], and so only a summary of the main results is provided here.

In the receptance approach, the overall structure is broken down at the joints into substructures, and the effect of the joints is introduced by applying sets of coupling forces. The fundamental model thus consists of substructures, each of which is subject to external driving forces and coupling forces, with these latter quantities then being the unk nowns that must be determined. This is achieved through the use of compatibility conditions at the joints, which allow a set of linear simultaneous equations to be set up that can then be solved and from which the coupling forces can be recovered.

To illustrate this approach, consider a plane network of beams which consists of $N$ beams and $M$ couplings. The beams have orientations $\theta$ in the global co-ordinate system. They äre assumed to be thin, long, of homogenous material and to have uniform cross-sections, so that Euler-Bernoulli theory is valid. Each beam $a$ has length $l_{a}$ and mass per unit length $\rho_{a}$. The damping $c_{a}$ is assumed to be viscous and proportional to mass. Let $\left\{Y_{a}^{\prime}\left(x_{1}^{\prime}\right)\right\}$ be the vector of displacements at the point $x_{1}^{\prime}$ of beam $a$ due to a vector of harmonic forces $\left\{F_{a}^{\prime}\left(x_{2}^{\prime}\right)\right\}$ which acts at the point $x_{2}^{\prime}$. The prime indicates that these quantities are specified in co-ordinates local to the beam. Green functions for this beam are then defined as follows

$$
\left\{\mathbf{Y}_{a}^{\prime}\left(x_{1}^{\prime}\right)\right\}=\left[\mathbf{G}_{a}^{\prime}\left(x_{1}^{\prime}, x_{2}^{\prime}\right)\right]\left\{\mathbf{F}_{a}^{\prime}\left(x_{2}^{\prime}\right)\right\},
$$

where

$$
\left[\mathbf{G}_{a}^{\prime}\left(x_{1}^{\prime}, \mathrm{x}_{2}^{\prime}\right)\right]=\left[\begin{array}{ccc}
G_{a, \text { }}^{\prime}\left(x_{1}^{\prime}, x_{2}^{\prime}\right) & 0 & 0 \\
0 & G_{a, v v}^{\prime}\left(x_{1}^{\prime}, x_{2}^{\prime}\right) & G_{a, v \theta}^{\prime}\left(x_{1}^{\prime}, x_{2}^{\prime}\right) \\
0 & G_{a, v v}^{\prime}\left(x_{1}^{\prime}, x_{2}^{\prime}\right) & G_{a, \theta \theta}^{\prime}\left(x_{1}^{\prime}, x_{2}^{\prime}\right)
\end{array}\right]
$$

The expressions for $G_{a, u u}^{\prime}\left(x_{1}^{\prime}, x_{2}^{\prime}\right), G_{a, v v}^{\prime}\left(x_{1}^{\prime}, x_{2}^{\prime}\right), G_{a, v \theta}^{\prime}\left(x_{1}^{\prime}, x_{2}^{\prime}\right), G_{a, \theta v}^{\prime}\left(x_{1}^{\prime}, x_{2}^{\prime}\right)$ and $G_{a, \theta \theta}^{\prime}\left(x_{1}^{\prime}, x_{2}^{\prime}\right)$ are available in closed forms for uniform beams for all boundary conditions. 


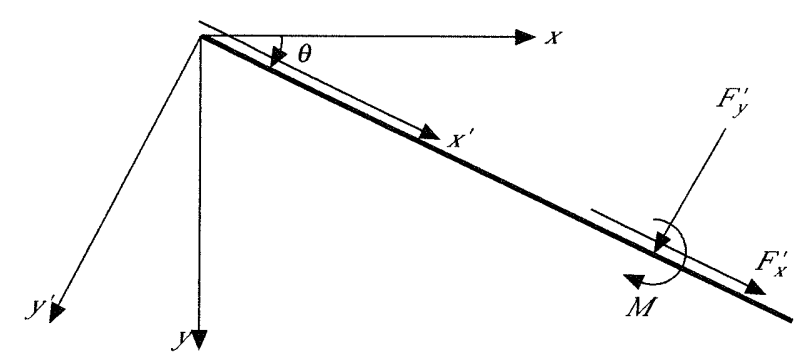

Figure 1. Local and global co-ordinates.

The vector of displacements of the beam in the global co-ordinates $\left\{\mathbf{Y}_{a}(x)\right\}$ is related to the vector of displacement in the local co-ordinates $\left\{\mathbf{Y}_{a}^{\prime}\left(x^{\prime}\right)\right\}$ through a transformation matrix $[\mathbf{T}]_{a}$ as follows:

$$
\left\{\mathbf{Y}_{a}^{\prime}\left(x^{\prime}\right)\right\}=[\mathbf{T}]_{a}\left\{\mathbf{Y}_{a}(x)\right\}
$$

where $[\mathbf{T}]$ is defined for any beam as

$$
[\mathbf{T}]=\left[\begin{array}{ccc}
\cos \theta & \sin \theta & 0 \\
-\sin \theta & \cos \theta & 0 \\
0 & 0 & 1
\end{array}\right]
$$

and $\theta$ is the angle between the local and global co-ordinates of the beam, as shown in Figure 1. A similar relationship exists between the vector of forces in the local and global co-ordinates, as follows:

$$
\left\{\mathbf{F}_{a}^{\prime}\left(x^{\prime}\right)\right\}=[\mathbf{T}]_{a}\left\{\mathbf{F}_{a}(x)\right\}
$$

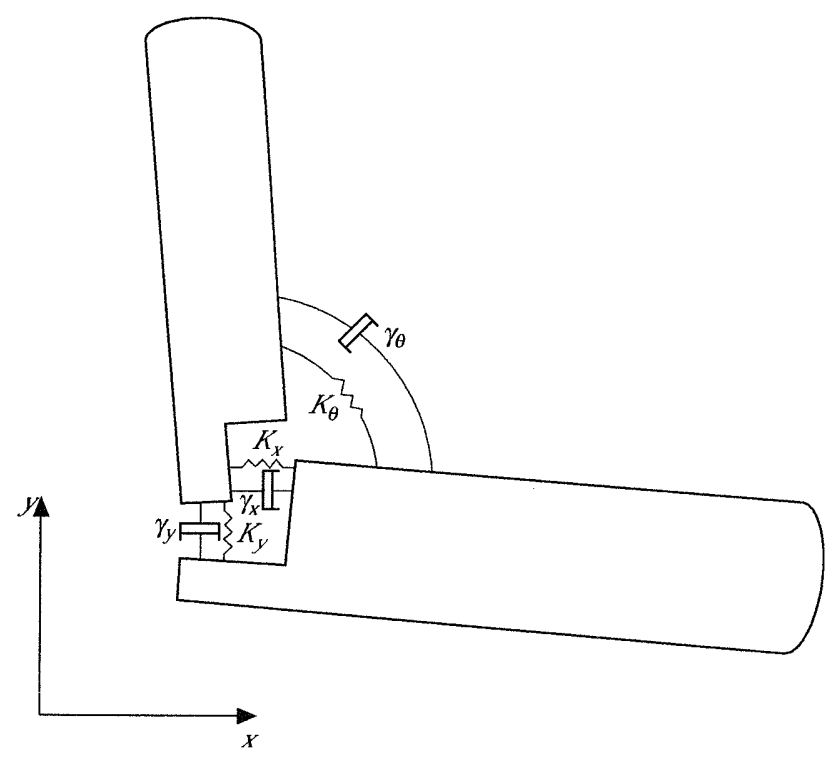

Figure 2. The joint model, showing coupling springs and dampers aligned to the global co-ordinate system 


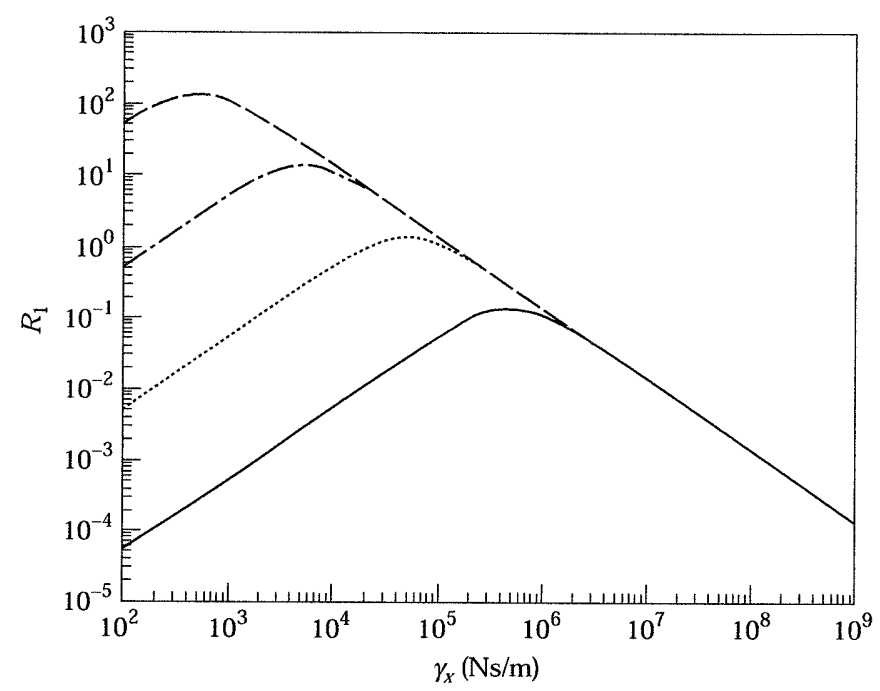

Figure 3. Variation in $R_{1}$ and $\gamma_{x}$ and $K_{x}$ for $\omega=10000 \mathrm{rad} / \mathrm{s}$ for the case of two beams at right angles coupled in the $x$ direction only. $-\cdots, K_{x}=5 \times 10^{6} \mathrm{~N} / \mathrm{m} ;-\cdots \cdots, K_{x}=5 \times 10^{7} \mathrm{~N} / \mathrm{m} ; \cdots, K_{x}=5 \times 10^{8} \mathrm{~N} / \mathrm{m} ;-$ $K_{x}=5 \times 10^{9} \mathrm{~N} / \mathrm{m}$.

Therefore, the matrix of Green functions in global co-ordinates is given by

$$
\left[\mathbf{G}_{a}\left(x_{1}, x_{2}\right)\right]=[\mathbf{T}]_{a}^{\mathrm{T}}\left[\mathbf{G}_{a}^{\prime}\left(x_{1}^{\prime}, x_{2}^{\prime}\right)\right][\mathbf{T}]_{a} .
$$

Here the joint between any two beams is assumed to be compliant and dissipative in the three degrees of freedom. Each joint is modelled by three springs of strengths $K_{x}, K_{y}$ and $K_{\theta}$, and three viscous dampers of strengths $\gamma_{x}, \gamma_{y}$ and $\gamma_{\theta}$ in the global $x, y$ and $\theta$ directions, respectively, as shown in Figure 2. Let the joint $i$ connect two beams, $a$ and $b$, at the points $\mathrm{A}$ and $\mathrm{B}$, respectively. The vectors of displacements of the two ends $\mathrm{A}$ and $\mathbf{B}$ are given, in global co-ordinates, as

$$
\{\mathbf{Y}\}_{\mathrm{A} i}=\left\{\begin{array}{l}
u_{\mathrm{A} i} \\
v_{\mathrm{A} i} \\
\theta_{\mathrm{A} i}
\end{array}\right\} \quad \text { and } \quad\{\mathbf{Y}\}_{\mathrm{B} i}=\left\{\begin{array}{c}
u_{\mathrm{B} i} \\
v_{\mathrm{B} i} \\
\theta_{\mathrm{B} i}
\end{array}\right\}
$$

Coupling forces between the beams arise due to the relative displacements between the two ends of the joint. If the dimensions of the joint are small compared to the length of the beams, then the vector of coupling forces which acts at the end $\mathrm{B}$ can be given as

$$
\{\mathbf{P}\}_{\text {coup }}=[\Omega]_{i}\left\{\{\mathbf{Y}\}_{\mathrm{A} i}-\{\mathbf{Y}\}_{\mathrm{B} i}\right\}=[\mathbf{\Omega}]_{i}\{\Delta \mathbf{Y}\}_{i},
$$

while the same forces act on the end $\mathrm{A}$ but in the opposite direction. $[\boldsymbol{\Omega}]_{i}$ is a diagonal matrix which has the joint complex stiffness as its diagonal elements. These coupling forces are the main unknowns in the receptance method that need to be determined. They are found through the introduction of suitable compatibility conditions. If $\{\mathbf{Y}\}_{\mathrm{A} 0}$ and $\{\mathbf{Y}\}_{\mathrm{B} 0}$ are the displacements of the points $A$ and $B$ of the beams when uncoupled and due to external forcing only, then superposition requires that the displacements at $\mathrm{A}$ and $\mathrm{B}$ of the coupled structure be equal to these displacements plus those due to the coupling forces which act 
at the joint. These conditions can be written as follows:

$$
\begin{aligned}
& \{\mathbf{Y}\}_{\mathrm{A}}=\{\mathbf{Y}\}_{\mathrm{A} 0}-[\mathbf{G}]_{\mathrm{A}}\{\mathbf{P}\}_{\text {coup }}=\{\mathbf{Y}\}_{\mathrm{A} 0}-[\mathbf{A}]_{\mathrm{A}}\{\Delta \mathbf{Y}\}, \\
& \{\mathbf{Y}\}_{\mathrm{B}}=\{\mathbf{Y}\}_{\mathrm{B} 0}+[\mathbf{G}]_{\mathrm{B}}\{\mathbf{P}\}_{\text {coup }}=\{\mathbf{Y}\}_{\mathrm{B} 0}+[\mathbf{A}]_{\mathrm{B}}\{\Delta \mathbf{Y}\},
\end{aligned}
$$

where

$$
\{\mathbf{A}\}_{\mathrm{A}}=[\mathbf{G}]_{\mathrm{A}}[\mathbf{\Omega}] \text { and }[\mathbf{A}]_{\mathrm{B}}=[\mathbf{G}]_{\mathrm{B}}[\mathbf{\Omega}] .
$$

From these last two equations, the vector of the relative displacements and therefore the vector of coupling forces can be written in terms of the vector of the relative displacements $\{\Delta \mathbf{Y}\}_{0}$ as follows:

$$
\{\Delta \mathbf{Y}\}=[\mathbf{D}]^{-1}\{\Delta \mathbf{Y}\}_{0} \quad \text { and } \quad\{\mathbf{P}\}_{\text {coup }}=[\mathbf{\Omega}][\mathbf{D}]^{-1}\{\Delta \mathbf{Y}\}_{0},
$$

where $[\mathbf{D}]$ is given by

$$
[\mathbf{D}]=[\mathbf{I}]+[\mathbf{A}]_{\mathrm{A}}+[\mathbf{B}]_{\mathrm{B}}
$$

Once the coupling forces are determined, it becomes possible to determine the displacements at any point in the system due to external forcing in terms of the displacements (and therefore Green functions) of the uncoupled systems. It should be noted that the dimension of the matrix [D] is $3 M$ and so depends only on the number of joints in the structure.

\subsection{ENERGY FLOWS AND DISSIPATION AT THE JOINTS}

The energy flow through a joint may be calculated from the product of force or moment and the velocity or angular velocity at the joint. The energy flow that enters joint $i$ at end A through the horizontal, vertical and rotational springs, for example, is found to be given by

$$
\{\boldsymbol{\Pi}\}_{\text {coup Ai }}=\sum_{a=1}^{3 N}\{\mathbf{H}\}_{\text {Aia }} S_{F_{a} F_{a}},
$$

where

$$
\begin{aligned}
& \{\mathbf{H}\}_{\text {Aia }}=\operatorname{Re}\left\{\mathrm{i} \omega[\mathbf{\Omega}]_{[}\left[\left([\mathbf{I}]+[\mathbf{A}]_{\mathrm{B}}\right)^{*} \mid[\mathbf{A}]_{\mathrm{A}}^{*}\right]\left[\begin{array}{cc}
{[\mathbf{D}]^{-1}} & {[\mathbf{0}]} \\
{[\mathbf{0}]} & {[\mathbf{D}]^{-1}}
\end{array}\right]\right]_{i}^{*} \\
& \left.\times[\mathbf{g} \mathbf{f}]_{a}^{*}[\mathbf{g}]_{a}^{T}\left[\left[\begin{array}{cc}
{[\mathbf{D}]^{-1}} & {[\mathbf{0}]^{-1}} \\
{[\mathbf{0}]} & {[\mathbf{D}]^{-1}}
\end{array}\right]^{\mathrm{T}}\left[[\mathbf{A}]_{\mathrm{B}} \mid(\mathbf{I} \mathbf{I}]+[\mathbf{A}]_{\mathrm{A}}\right)\right]^{\mathrm{T}}\right]_{i} \\
& \left.+[\gamma]_{i} \omega^{2}\left[\left([\mathbf{I}]+[\mathbf{A}]_{\mathrm{B}}\right)^{*} \mid[\mathbf{A}]_{\mathrm{A}}^{*}\right]\left[\left[\begin{array}{cc}
{[\mathbf{D}]^{-1}} & {[\mathbf{0}]} \\
{[\mathbf{0}]} & {[\mathbf{D}]^{-1}}
\end{array}\right]^{*}\right]\right]_{i} \\
& \left.\times[\mathbf{g} \mathbf{f}]_{a}^{*}[\mathbf{g} \mathbf{f}]_{a}^{T}\left[\left[\begin{array}{cc}
{[\mathbf{D}]^{-1}} & {[\mathbf{0}]} \\
{[\mathbf{0}]} & {[\mathbf{D}]^{-1}}
\end{array}\right]^{\mathrm{T}}\left[\left([\mathbf{I}]+[\mathbf{A}]_{\mathrm{B}}\right) \mid[\mathbf{A}]_{\mathrm{A}}\right]^{\mathrm{T}}\right]\right\},
\end{aligned}
$$

where the matrix [gf] contains the integrals of the Green functions and forces taken over the individual subsystems and $S_{F_{0} F_{a}}$ is the spectrum of the forcing acting on a subsystem, which is assumed to be spatially incoherent and also incoherent between subsystems. The 
energy flow that enters joint $i$ at end B through the horizontal, vertical and rotational springs is derived similarly.

The energy dissipated at the joint is then deduced from an energy balance at the joint as follows:

$$
\{\boldsymbol{\Pi}\}_{\mathrm{DC} i}=\{\boldsymbol{\Pi}\}_{\text {coup } \mathrm{A} i}+\{\boldsymbol{\Pi}\}_{\text {coup } \mathrm{B} i}
$$

Therefore

$$
\{\boldsymbol{\Pi}\}_{\mathrm{DC} i}=\sum_{a=1}^{3 N}\{\mathbf{H}\}_{\mathrm{DCia}} S_{F_{a} F_{a}}
$$

where $[\mathbf{H}]_{\mathrm{DCia}}$ denotes the energy dissipated at joint $i$ due to forcing on subsystem $a$ and is given by

$$
\{\mathbf{H}\}_{\mathrm{DC} i a}=\{\mathbf{H}\}_{\mathrm{A} i a}+\{\mathbf{H}\}_{\mathrm{B} i a}
$$

\subsection{INPUT POWER}

The input power due to forcing on subsystem $a$ is calculated from the diagonal elements of the product of the vector of force applied to the subsystem and the vector at the point of application. The input power into subsystem $a$ is found to be given by

$$
\{\boldsymbol{\Pi}\}_{\mathrm{IN} a}=[\mathbf{H}]_{\mathrm{IN} a, d i a g}\left[\mathbf{S}_{F_{a} F_{a}}\right]
$$

where the input power receptance is given by

$$
\begin{aligned}
{[\mathbf{H}]_{\mathrm{IN} a}=} & -\omega \operatorname{Im}\left\{\iint_{a}\left[\mathbf{G}_{a}\left(x_{i}, x_{j}\right) \mathbf{f}_{a a}\left(x_{i}, x_{j}\right)\right] \mathrm{d} x_{i} \mathrm{~d} x_{j}\right. \\
& \pm \sum_{l=1}^{M} \sum_{m=1}^{M} \iint_{a}\left[\left[\mathbf{G}_{a}\left(x, x_{l}\right)\right]\left[[\mathbf{\Omega}]_{l}[\mathbf{D}]_{l i m}^{-1}\left[\mathbf{G}_{a}\left(x_{m}, y\right)\right]\left[\mathbf{f}_{a a}(x, y)\right] \mathrm{d} x \mathrm{~d} y\right\}\right.
\end{aligned}
$$

and $f_{a a}$ describes the spatial variation of the driving force.

\subsection{ENERGY LEVELS}

If the vector of energies leaving the subsystems is denoted by $[\mathbf{\Pi}]_{\text {ouT }}$, then this vector can be related to vector of energies flowing through the coupling systems at the ends $A$

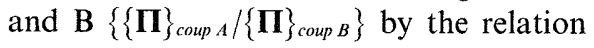

$$
\{\boldsymbol{\Pi}\}_{\text {ouT }}=[\mathbf{C O N}]\left\{\frac{\{\boldsymbol{\Pi}\}_{\text {coup } \mathrm{A}}}{\{\boldsymbol{\Pi}\}_{\text {coup } \mathrm{B}}}\right\}
$$

where the elements of the connectivity matrix [CON] are either 1 or 0 and define the topology of the overall system. Hence the energy dissipated within each subsystem due to damping can be easily deduced from the energy balance for each subsystem as follows:

$$
\{\boldsymbol{\Pi}\}_{\text {Diss }}=\{\boldsymbol{\Pi}\}_{\text {IN }}-\{\boldsymbol{\Pi}\}_{\text {out }} .
$$

Finally, the energy levels for each subsystem can be related to the energy dissipated due to damping by the well known relationship

$$
\{\mathbf{E}\}=[\mathbf{c}]^{-1}\{\boldsymbol{\Pi}\} \text { DIss, }
$$


where $[\mathbf{c}]$ is the diagonal matrix of damping constants of each subsystem, and the damping of each subsystem is assumed to be viscous and proportional to mass per unit length.

\section{EXAMPLES}

\subsection{ENERGY RECEPTANCES}

It is commonly the case that the joints in a built-up structure dissipate more energy than material damping does [10]. It is therefore of interest to see the effects of variations in joint damping on the magnitudes of the energy transferred, or dissipated within, each subsystem.

Consider two free-free beams at right angles connected together by three springs and three dampers in the three degrees of freedom. Beam 1 is horizontal and beam 2 is vertical; the parameters adopted for this supply are given below in Table 1 (case $b$, low bending rigidity). The first beam is excited by a harmonic force at the end away from the joint. Let $Z_{x}=K_{x} / \omega+\mathrm{i} \gamma_{x}$ be the joint impedance in the $x$ direction, $Z_{y}=K_{y} / \omega+\mathrm{i} \gamma_{y}$ be the joint impedance in the $y$ direction and $Z_{\theta}=K_{\theta} / \omega+\mathrm{i} \gamma_{\theta}$ be the joint impedance in the $\theta$ direction. If $C_{\mathrm{B}}$ represents the bending wave speed, the dimensionless ratios $\hat{Z}_{x}=Z_{x} /\left(\rho_{2} C_{\mathrm{B} 2}\right)$, $\hat{Z}_{y}=Z_{y} /\left(\rho_{1} C_{\mathrm{BI}}\right)$ and $\hat{Z}_{\theta}=\left(C_{\mathrm{B} 1} Z_{\theta}\right) / E I_{1}$ are then the ratios of the bending wave impedances and the impedances of the joint. In what follows, $R_{1}$ denotes the ratio of the power dissipated at the joint to the power transferred to the undriven beam and $R_{2}$ denotes the ratio of the power dissipated at the joint to the power input by the external forcing. These ratios are very small when the joint damping is either very weak or very strong: in the first case, because the damper is too weak to have much effect and, in the second, because the damper is so stiff that it is hardly deflected [20]. It is therefore expected that these ratios are maximized for certain levels of the coupling damping.

\subsubsection{Two beams at right angles coupled in the $x$ direction only}

First; beam 1 is driven by a horizontal (axial) force at the end away from the coupling which, being only in the $x$ direction, excites just the flexural modes of beam 2 . The expressions for the energy receptances in this case can be given as follows:

$$
\begin{gathered}
H_{\mathrm{B} 11}=-\omega \frac{\left|\Omega_{x}\right|^{2}}{|D|^{2}} \operatorname{Im}\left\{G_{2, v v}^{\prime}\left(x_{\mathrm{B}}^{\prime}, x_{\mathrm{B}}^{\prime}\right)\right\}\left|G_{1, u u}^{\prime}\left(x_{0}^{\prime}, x_{0}^{\prime}\right)\right|^{2}, \\
H_{\mathrm{DC}}=\frac{\gamma_{x} \omega^{2}}{|D|^{2}}\left|G_{1, u u}^{\prime}\left(x_{0}^{\prime}, x_{0}^{\prime}\right)\right|^{2}
\end{gathered}
$$

and

$$
H_{\mathrm{IN} 1}=-\omega \operatorname{Im}\left\{G_{1, u u}^{\prime}\left(x_{0}^{\prime}, x_{0}^{\prime}\right)-\frac{\Omega_{x}}{D}\left(G_{\mathrm{l}, u u}^{\prime}\left(x_{\mathrm{A}}^{\prime}, x_{0}^{\prime}\right)\right)^{2}\right\},
$$

where

$$
D=1+\Omega_{x}\left(G_{1, u u}^{\prime}\left(x_{\mathrm{A}}^{\prime}, x_{\mathrm{A}}^{\prime}\right)+G_{2, v v}^{\prime}\left(x_{\mathrm{B}}^{\prime}, x_{\mathrm{B}}^{\prime}\right)\right) \text {. }
$$

From these expressions it can be shown that the ratio $R_{1}$ increases as $\gamma_{x}$ increases, until it reaches a maximum level when $\gamma_{x}=K_{x} / \omega$, after which it begins to fall again: see Figure 3 . This figure also shows that for a weak spring the ratio $R_{1}$ is at high levels and drops as the spring strength increases; until it becomes very low for strong springs, as might be expected. In Figure 4 is shown the variation of the ratio $R_{2}$ with the damper strength $\gamma_{x}$ for increasing values of the spring strength $K_{x}$ while the frequency $\omega$ is constant. It is seen that for a very strong spring, the maximum ratio $R_{2}$ also occurs when $\gamma_{2}=K_{x} / \omega$. However, 


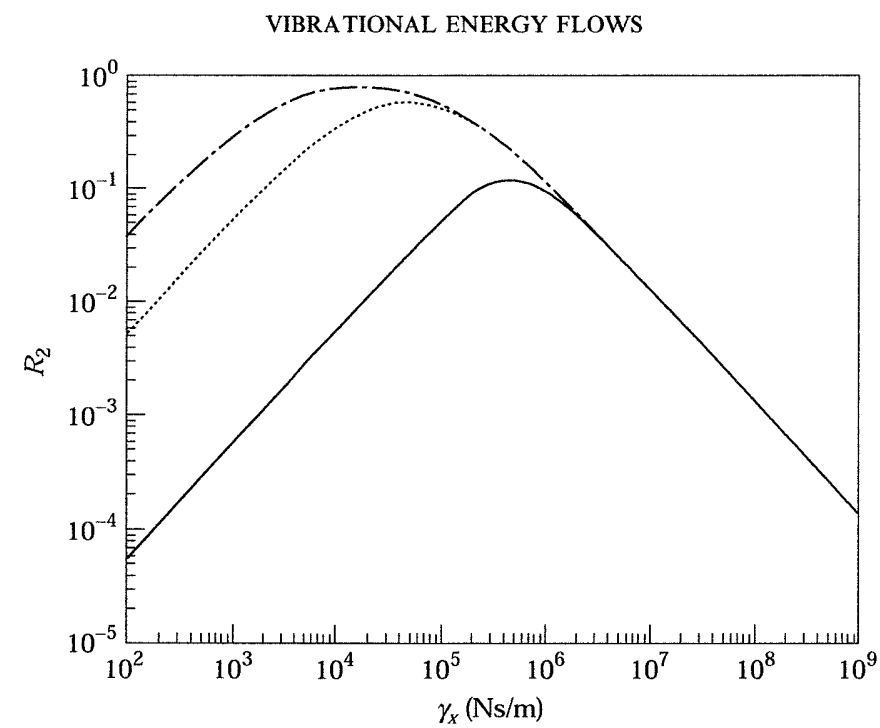

Figure 4. Variation in $R_{2}$ with $\gamma_{x}$ and $K_{x}$ for $\omega=10000 \mathrm{rad} / \mathrm{s}$ for the case of two beams at right angles coupled in the $x$ direction only: key as Figure 3 (curves for $K_{x}=5 \times 10^{6} \mathrm{~N} / \mathrm{m}$ and $K_{x}=5 \times 10^{7} \mathrm{~N} / \mathrm{m}$ superimposed).

it is then very small because the damper is virtually blocked and unable to dissipate much energy. For the case of a weak spring, the ratio $R_{2}$ is higher but becomes maximum at values $\gamma_{x}$ that depend on the spring stiffness (and also the frequency).

In Figure 5 is shown the variation of the ratio $R_{2}$ with the driving frequency $\omega$ for increasing values of the damper strength $\gamma_{x}$ while the spring stiffness is constant and weak. It transpires that $R_{2}$ always drops at the flexural natural frequencies of the undriven beam, because the absolute values of the dissipated power at the joint is at minimum level at these frequencies, since $D$-which occurs solely in the denominator of equation (25)-is then

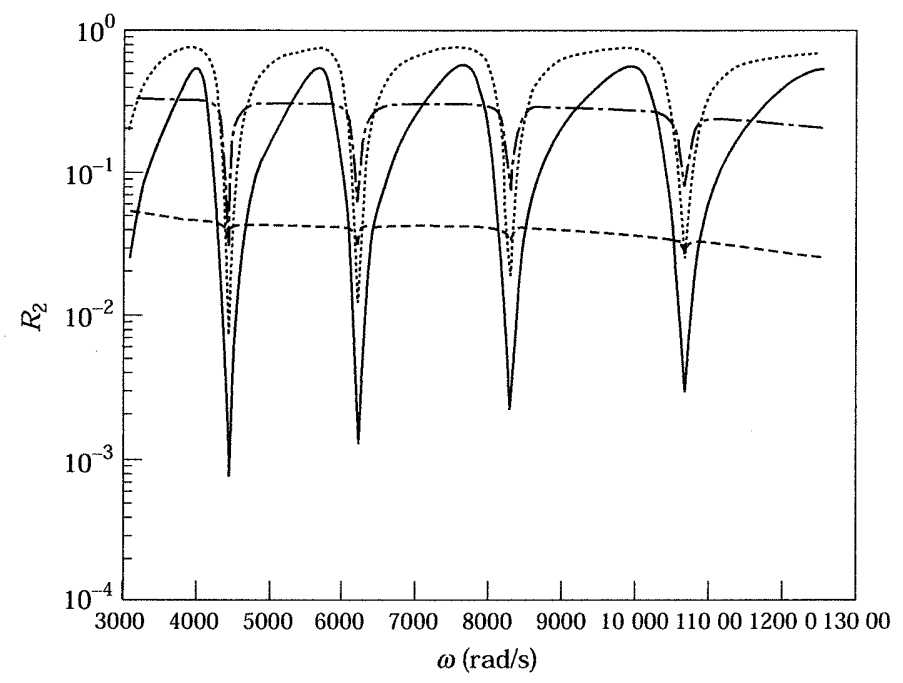

Figure 5. Variation in $R_{2}$ with $\omega$ and $\gamma_{x}$ for $K_{x}=10^{5} \mathrm{~N} / \mathrm{m}$ for the case of two beams at right angles coupled in the $x$ direction only. - - - $\gamma_{x}=10^{2} \mathrm{Ns} / \mathrm{m} ; \cdots \cdots+-, \gamma_{x}=10^{3} \mathrm{Ns} / \mathrm{m} ; \cdots, \gamma_{x}=10^{4} \mathrm{Ns} / \mathrm{m} ;-\cdots, \gamma_{x}=10^{5} \mathrm{Ns} / \mathrm{m}$. 


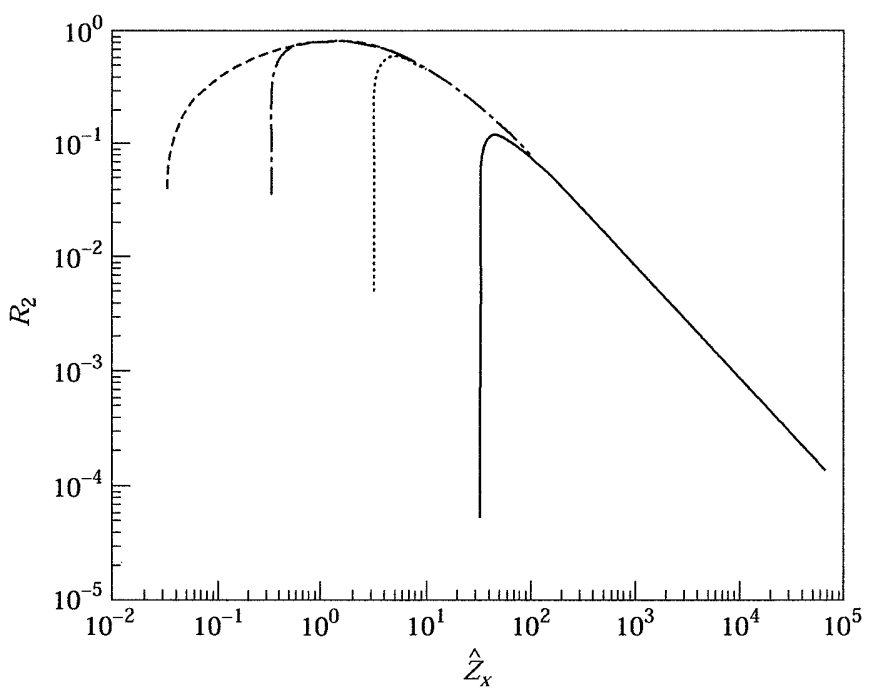

Figure 6. Variation in $R_{2}$ with the impedance ratio $\hat{Z}_{x}$ an d $K_{x}$ for $\omega=10000 \mathrm{rad} / \mathrm{s}$ for the case of two beams at right angles coupled in the $x$ direction only: key as Figure 3 .

maximized (see equation (27)). In Figure 6 is shown the variation of the ratio $R_{2}$ versus the impedance ratio $\hat{Z}_{x}$ when the damper strength is varied for a constant value of the driving frequency $\omega$. It is clear that the value of the maximum ratio $R_{2}$ depends on the value of the spring strength $K_{x}$ and that for a weak spring this maximum ratio yields impedance ratios $\hat{Z}_{x}$ close to unity.

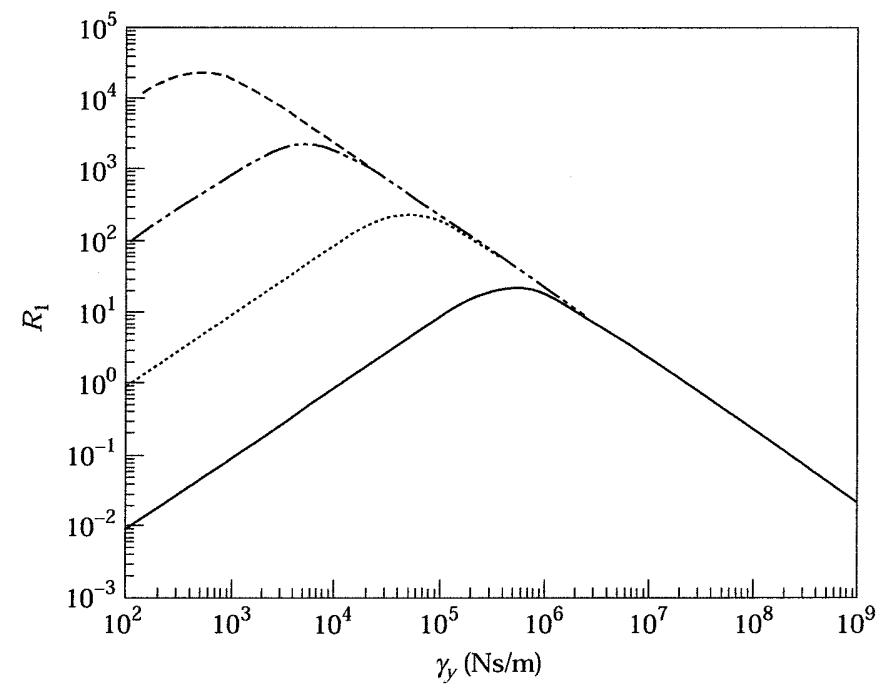

Figure 7. Variation in $R_{1}$ with $\gamma_{s}$ and $K_{y}$ for $\omega=10000 \mathrm{rad} / \mathrm{s}$ for the case of two beams at right angles coupled in the $y$ direction only. $\cdots, K_{y}=5 \times 10^{6} \mathrm{~N} / \mathrm{m} ;-\cdot-\cdot-, K_{y}=5 \times 10^{7} \mathrm{~N} / \mathrm{m} ; \cdots, K_{r}=5 \times 10^{8} \mathrm{~N} / \mathrm{m} ;-$ $K_{\mathrm{y}}=5 \times 10^{9} \mathrm{~N} / \mathrm{m}$. 


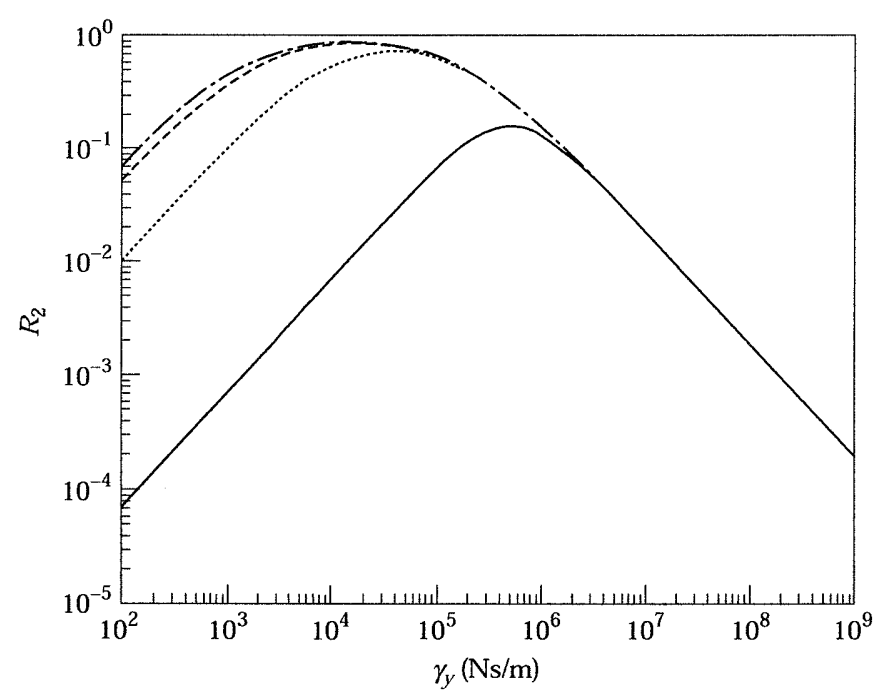

Figure 8. Variation in $R_{2}$ with $\gamma_{y}$ and $K_{y}$ for $\omega=10000 \mathrm{rad} / \mathrm{s}$ for the case of two beams at right angles coupled in the $y$ direction only: key as Figure 7 .

\subsubsection{Two beams at right angles coupled in the $y$ direction only}

In this case beam 1 is driven by a vertical (transverse) load and the coupling, being only in the $y$ direction, excites just the longitudinal modes in beam 2 . The expressions for the energy receptances in this case are

$$
\begin{gathered}
H_{\mathrm{B} 11}=-\omega \frac{\left|\Omega_{y}\right|^{2}}{|D|^{2}} \operatorname{Im}\left\{G_{2, u u}^{\prime}\left(x_{\mathrm{B}}^{\prime}, x_{\mathrm{B}}^{\prime}\right)\right\}\left|G_{1, v v}^{\prime}\left(x_{0}^{\prime}, x_{0}^{\prime}\right)\right|^{2}, \\
H_{\mathrm{DC}}=\frac{\gamma_{y} \omega^{2}}{|D|^{2}}\left|G_{1, v v}^{\prime}\left(x_{0}^{\prime}, x_{0}^{\prime}\right)\right|^{2}
\end{gathered}
$$

and

$$
H_{\mathrm{INI}}=-\omega \operatorname{Im}\left\{G_{1, v v}^{\prime}\left(x_{0}^{\prime}, x_{0}^{\prime}\right)-\frac{\Omega_{y}}{D}\left(G_{1, v v}^{\prime}\left(x_{\mathrm{A}}^{\prime}, x_{0}^{\prime}\right)\right)^{2}\right\}
$$

where

$$
D=1+\Omega_{y}\left(G_{1, v v}^{\prime}\left(x_{\mathrm{A}}^{\prime}, x_{\mathrm{A}}^{\prime}\right)+G_{2, u u}^{\prime}\left(x_{\mathrm{B}}^{\prime}, x_{\mathrm{B}}^{\prime}\right)\right) .
$$

As in the previous case, it can be shown from these expressions that the ratio $R_{\mathrm{f}}$ increases as $\gamma_{y}$ increases until it reaches a maximum level when $\gamma_{y}=K_{y} / \omega$, after which it begins to fall again: see Figure 7. This figure also shows that for a weak spring the ratio $R_{1}$ is at high levels and drops as the springs strength increases, again as might be expected. In Figure 8 is shown the variation of the ratio $R_{2}$ with the damper strength $\gamma_{y}$ for increasing values of the spring strength $K_{y}$ while the frequency $\omega$ is constant. Again, it is seen that for a very strong spring, the maximum ratio $R_{2}$ occurs when $\gamma_{y}=K_{y} / \omega$ but is then very small. For the case of a weak spring, the ratio $R_{2}$ takes higher levels, but once more becomes maximum at values of $\gamma_{p}$ which depend on the spring stiffness and frequency.

In Figure 9 is shown the variation of the ratio $R_{2}$ with the driving frequency $\omega$ for different values of the damper strength $\gamma_{y}$ while the spring stiffness is constant and weak. 


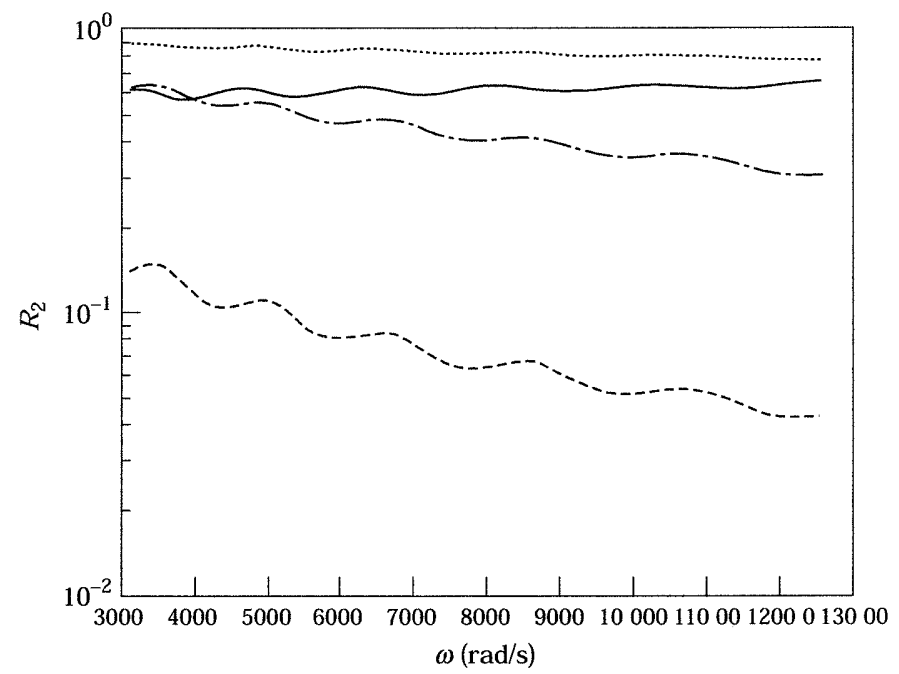

Figure 9. Variation in $R_{2}$ with $\omega$ and $\gamma_{y}$ for $K_{y}=10^{5} \mathrm{~N} / \mathrm{m}$ for the case of two beams at right angles coupled in the $y$ direction only: key as Figure 5 .

Here again the ratio $R_{2}$ is maximized for certain values of $\gamma_{y}$ and the dissipated power at the junction is then $80-90 \%$ of the input power. This means that by the careful selection of the damper strength, given a suitable coupling spring, it is possible to arrange for the bulk of the input power to be dissipated at the damper, an idea which is employed in many vibration control problems. Note that because the natural frequencies of the axial modes of the undriven beam lie well beyond the frequency range of interest here, $R_{2}$ does not show any marked dips, instead varying rather smoothly over the frequency range of interest.

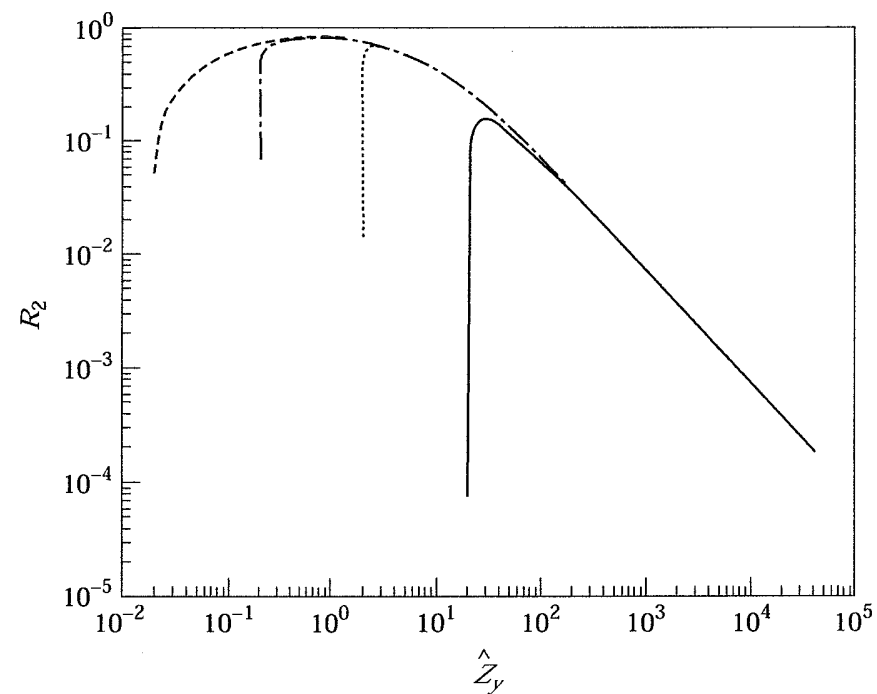

Figure 10. Variation in $R_{2}$ with the impedance ratio $\hat{Z}_{y}$ and $K_{y}$ for $\omega=10000 \mathrm{rad} / \mathrm{s}$ for the case of two beams at right angles coupled in the $y$ direction only: key as Figure 7 . 


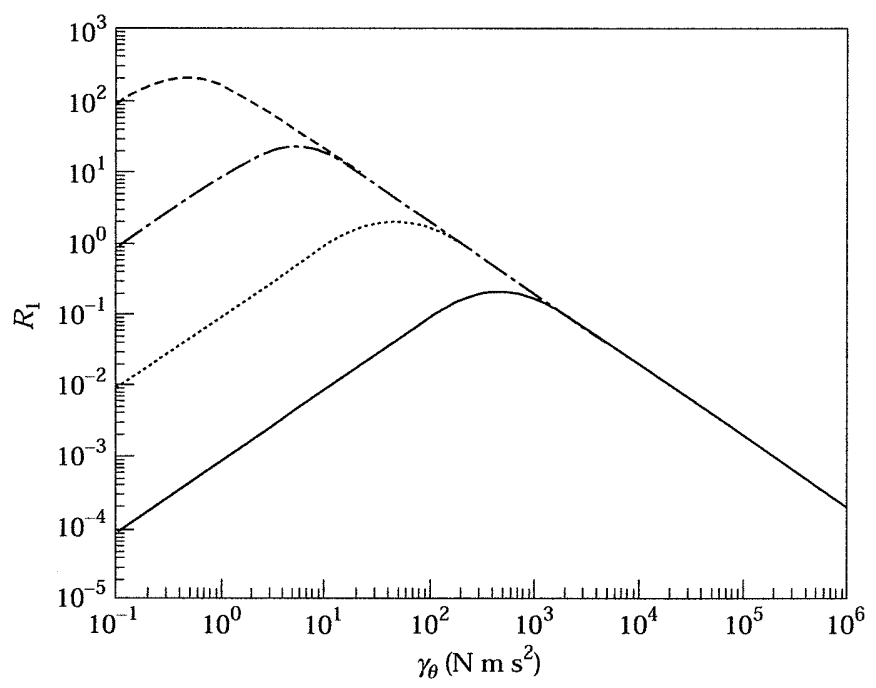

Figure 11. Variation in $R_{1}$ with $\gamma_{0}$ and $K_{0}$ for $\omega=10000 \mathrm{rad} / \mathrm{s}$ for the case of two beams at right angles coupled through the rotational spring only. $\cdots, K_{0}=5 \times 10^{3} \mathrm{~N} \mathrm{~s} \mathrm{~m} ;-\cdots \cdots, K_{0}=5 \times 10^{4} \mathrm{~N} \mathrm{~s} \mathrm{~m} ; \cdots$ $K_{0}=5 \times 10^{5} \mathrm{~N} \mathrm{~s} \mathrm{~m} ;-, K_{0}=5 \times 10^{6} \mathrm{~N} \mathrm{~s} \mathrm{~m}$.

When the ratio $R_{2}$ is plotted against $\hat{Z}_{y}$, the result is similar to that in Figure 6 , which shows that for a weak spring the optimum value of $\gamma_{y}$ is again associated with a value of $\hat{Z}_{y}$ in the proximity of unity: see Figure 10 .

\subsubsection{Two beams at right angles coupled through a rotational spring only}

Next, beam 1 is driven by a moment and the coupling again excites only the flexural modes in beam 2 . The expressions for the energy receptances in the case are

$$
\begin{gathered}
H_{\mathrm{B} I 1}=-\omega \frac{\left|\Omega_{\theta}\right|^{2}}{|D|^{2}} \operatorname{Im}\left\{G_{2, \theta \theta}^{\prime}\left(x_{\mathrm{B}}^{\prime}, x_{\mathrm{B}}^{\prime}\right)\right\}\left|G_{1, \theta \theta}^{\prime}\left(x_{0}^{\prime}, x_{0}^{\prime}\right)\right|^{2}, \\
H_{\mathrm{DC}}=\frac{\gamma_{\theta} \omega^{2}}{|D|^{2}}\left|G_{1, \theta \theta}^{\prime}\left(x_{0}^{\prime}, x_{0}^{\prime}\right)\right|^{2}
\end{gathered}
$$

and

$$
H_{\mathrm{IN} 1}=-\omega \operatorname{Im}\left\{G_{1, \theta \theta}^{\prime}\left(x_{0}^{\prime}, x_{0}^{\prime}\right)-\frac{\Omega_{\theta}}{D}\left(G_{1, \theta \theta}^{\prime}\left(x_{\mathrm{A}}^{\prime}, x_{0}^{\prime}\right)\right)^{2}\right\}
$$

where

$$
D=1+\Omega_{\theta}\left(G_{1, \theta \theta}^{\prime}\left(x_{\mathrm{A}}^{\prime}, x_{\mathrm{A}}^{\prime}\right)+G_{2, \theta \theta}^{\prime}\left(x_{\mathrm{B}}^{\prime}, x_{\mathrm{B}}^{\prime}\right)\right) .
$$

Here also, the ratio $R_{1}$ increases as $\gamma_{\theta}$ increases, until it reaches a maximum level when $\gamma_{\theta}=K_{\theta} / \omega$ and then begins to fall again: see Figure 11. It is also smallest for a strong spring. The variation of the ratio $R_{2}$ with the damper strength has a behaviour similar to that in the previous two cases: see Figure 12. In Figure 13 is shown the variation of the ratio with the driving frequency $\omega$ for increasing values of the damper strength $\gamma_{\theta}$ while the spring stiffness is constant and weak. It is seen that $R_{2}$ drops at the flexural natural frequencies 


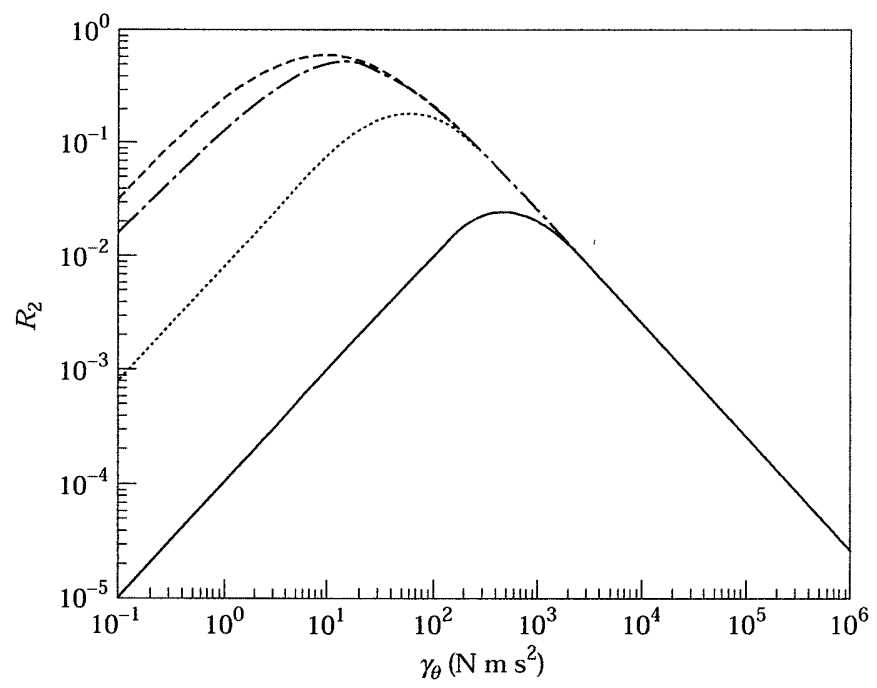

Figure 12. Variation in $R_{2}$ with $\gamma_{0}$ and $K_{0}$ for $\omega=10000 \mathrm{rad} / \mathrm{s}$ for the case of two beams at right angles coupled through the rotational spring only: key as Figure 11 .

of the undriven beam, which is similar to the case of two beams coupled in the $x$ direction only.

\subsection{VARIATION OF ENERGY FLOW WITH THE ANGLE BETWEEN TWO BEAMS COUPLED} TOGETHER BY A SET OF THREE SPRINGS

The final case considered here deals with two free-free beams coupled by three springs, where the coupling is conservative and weak. The first beam is excited by a harmonic force

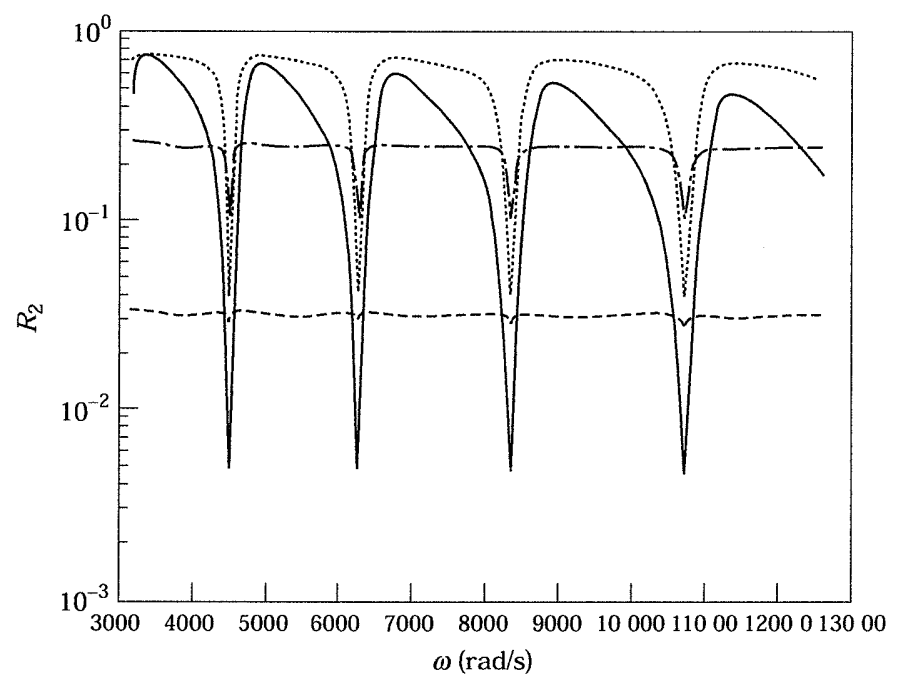

Figure 13. Variation in $R_{2}$ with $\omega$ and $\gamma_{0}$ for $K_{0}=100 \mathrm{~N} \mathrm{~s} \mathrm{~m}$ for the case of two beams at right angles coupled through the rotational spring only. $\cdots, \gamma_{0}=0.1 \mathrm{~N} \mathrm{~m} \mathrm{~s}^{2} ; \ldots \ldots, \gamma_{0}=1 \mathrm{~N} \mathrm{~m} \mathrm{~s}^{2} ; \cdots, \gamma_{0}=10 \mathrm{~N} \mathrm{~m} \mathrm{~s}^{2} ;-$ $\gamma_{0}=100 \mathrm{~N} \mathrm{~m} \mathrm{~s}^{2}$. 


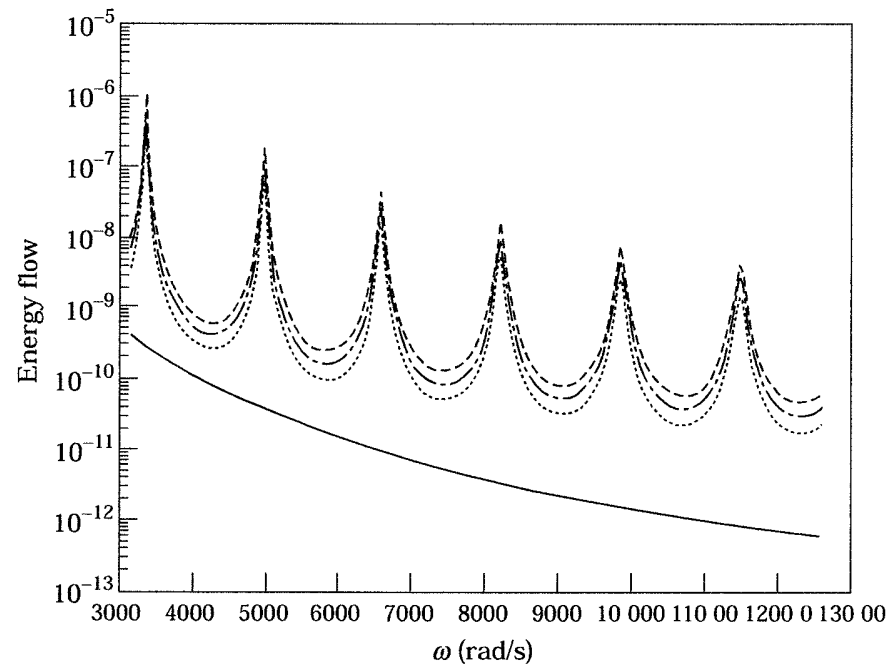

Figure 14. Variation in energy flow with $\omega$ and the angle between the two beams when energy transfer is dominated by axial modes and the first beam is subject to a vertical (tranverse) load (case a): $K_{x}=K_{y}=10^{7} \mathrm{~N} / \mathrm{m}$,

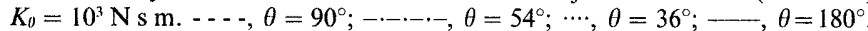

at the end away from the coupling. Two sets of physical parameters are used: in the first the bending stiffness is large so that the modal density of flexural modes is small compared to that of axial modes; and in the second the reverse is true and there are then more flexural modes than axial modes. These properties are detailed in Table 1 . The angle between the beams, $\theta$, takes the values $36^{\circ}, 54^{\circ}, 90^{\circ}$ and $180^{\circ}$, with beam 1 being kept horizontal throughout.

In the first set of examples, beam 1 is excited by a vertical (transverse) load. It is noted that for the case of two beams in parallel, the energy flow is entirely due to the flexural modes. When the bending stiffness is large, so that the modal density of the flexural modes is small (case a), the energy flow is then at minimum levels. As $\theta$ increases, the energy flow increases and reaches maximum levels when the beams are at right angles. In this case the axial modes are responsible for the bulk of energy flow with the peaks occurring at the natural frequencies of the axial modes of the undriven beam, as might be expected: see Figure 14. This result is in agreement with that shown by Horner and White [21], which

TABLE 1

The parameters used in the examples

\begin{tabular}{lcccl}
\hline \multicolumn{1}{c}{ Parameter } & Beam 1 & Beam 2 & Units \\
\hline Mass density, $\rho$ & & 56.16 & 39.78 & $\mathrm{~kg} / \mathrm{m}$ \\
Length, $l$ & 1.200 & 1.00 & $\mathrm{~m}$ \\
Damping strength, $\eta$ & & 0.01 & 0.01 & - \\
Rigidity, $E A$ & Case a & $1.512 \times 10^{7}$ & $1.071 \times 10^{9}$ & $\mathrm{~N}$ \\
& Case b & $1.512 \times 10^{9}$ & $1.071 \times 10^{9}$ & $\mathrm{~N}$ \\
Bending rigidity, $E I$ & Case a & $18.144 \times 10^{7}$ & $8.925 \times 10^{3}$ & $\mathrm{Nm}^{2}$ \\
& Case b & $18.144 \times 10^{3}$ & $8.925 \times 10^{3}$ & $\mathrm{Nm}^{2}$ \\
\hline
\end{tabular}




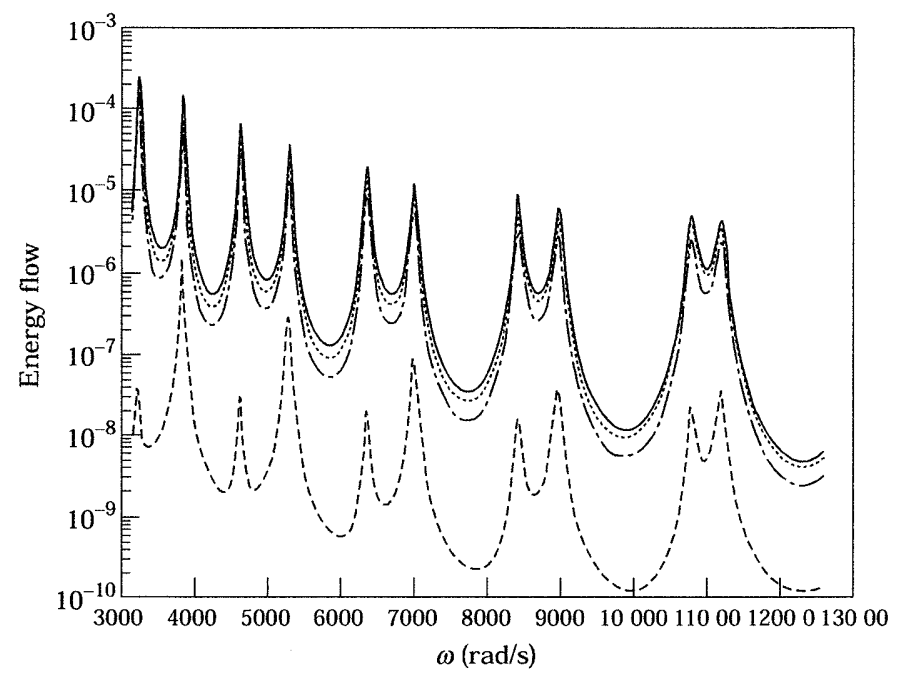

Figure 15. Variation in energy flow with $\omega$ and the angle between the two beams when energy transfer is dominated by longitudinal modes and the first beam is subject to a vertical (transverse) load (case b): key as Figure 14.

was derived using semi-infinite beams and a wave approach. On the other hand, when the modal density of the flexural modes is large (case b), the energy flow when the beams are parallel is entirely dominated by the flexural modes (with peaks at their natural frequencies). For other values of $\theta$, the axial modes then have very little effect on the overall energy flow. Moreover, as $\theta$ increases the energy flow decreases, and reaches minimum levels when the beams are at right angles: see Figure 15.

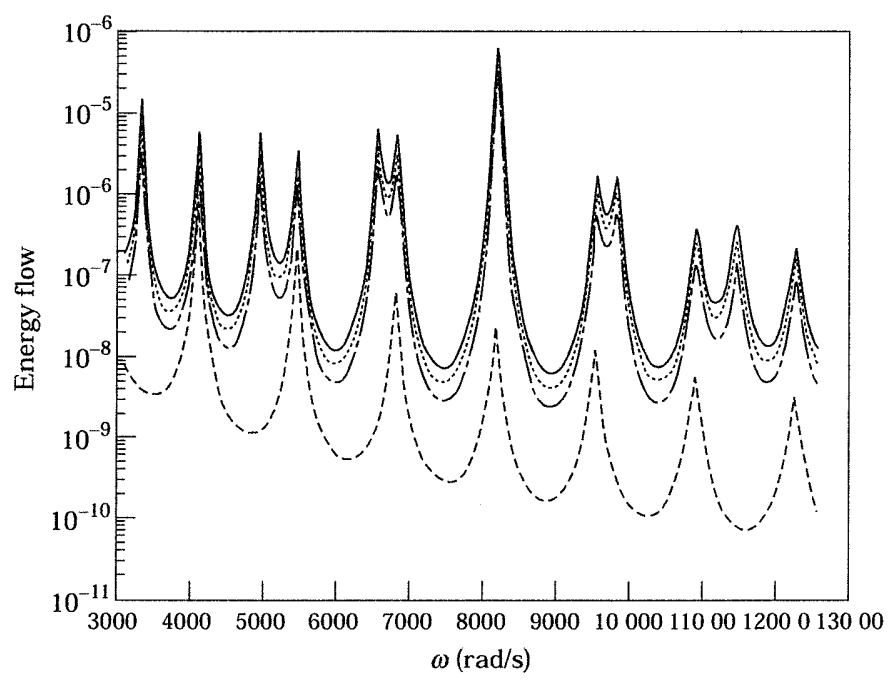

Figure 16. Variation in energy flow with $\omega$ and the angle between the two beams when energy transfer is dominated by axial modes and the first beam is subject to a horizontal (axial) load (case a): key as Figure 14 . 


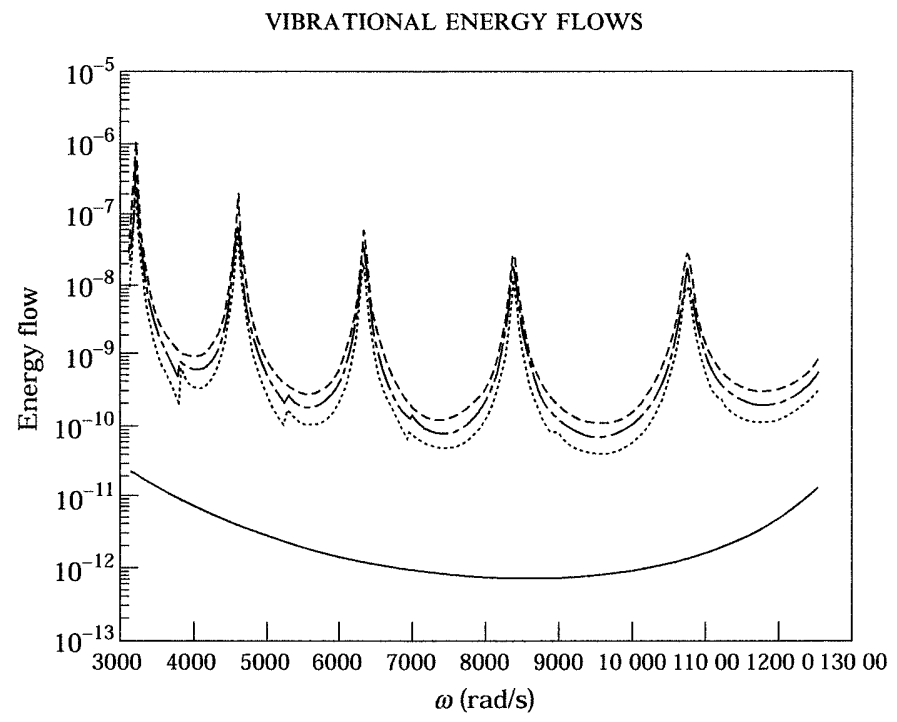

Figure 17. Variation in energy flow with $\omega$ and the angle between the two beams when energy transfer is dominated by longitudinal modes and the first beam is subject to a horizontal (axial) load (case b): key as Figure 14.

In the second set of examples, beam 1 is excited by a horizontal (axial) force. When the beams are parallel the axial modes transfer all the energy through the horizontal spring with peaks at the natural frequencies of the axial modes of the two beams. When the modal density of the axial modes is large compared to the modal density of the flexural modes (case a) the energy flow is maximum when the two beams are in parallel. It then decreases as the angle $\theta$ increases, until it reaches minimum values when the two beams are at right angles with peaks at the natural frequencies of the flexural modes of the undriven beam: see Figure 16. This result also agrees well with those given by Horner and White [21] for semi-infinite beams. In this set of examples when the modal density of the axial modes is small compared to the modal density of the flexural modes (case b), the energy flow is minimum for the case of two beams in parallel. It then rises as the angle increases, until it reaches maximum levels when the beams are at right angles, with peaks at the natural frequencies of the flexural modes of the undriven system: see Figure 17.

Note that in all of these cases the peaks in the curves occur at the natural frequencies of the underlying subsystems, because the couplings are weak and their resonances are then little affected by the action of the couplings.

\section{CONCLUSIONS}

Exact expressions for the energy flows through a plane network of beams have been derived based on a receptance approach, assuming the joints to be compliant and dissipative, and where each is modelled by a spring and viscous damper. An example consisting of two beams at right angles has then been considered, and the dissipation of power at the joint studied when the beams are connected throught the $x, y$ and rotational degrees of freedom, respectively. It is shown that the ratio of the dissipated power at the joint to the energy transmitted to the undriven subsystem is maximized at certain values of the damper strength which depend on the driving frequency and the coupling spring strength. This ratio is small for either small or large values of the coupling damping. By 
selecting the correct value of coupling damping, it is possible to arrange for most of the energy injected into the system to be dissipated in the joint. However, when the coupling spring is stiff, only a small amount of energy is dissipated at the joint regardless of the damper strength, as might be expected. It is also shown that in the case of weak coupling, the wave impedance to joint impedance ratio is a good measure of when the joint is able to dissipate maximum energy.

The variation of energy flow through a joint with the angle between two beams has also been studied. It is shown that it is heavily dependent on the longitudinal to flexural modal density ratio of the connected beams. If the flow of energy is dominated by the longitudinal modes (high longitudinal modal density compared to flexural modal density), then the energy flow due to a flexural incident wave will be a minimum when the beams are in parallel and will increase until it becomes a maximum when the beams are at right angles. Conversely, the energy flow due to a longitudinal incident wave is a maximum when the beams are in parallel and decreases until it is minimized when the beams are at right angles. On the other hand, when the flexural modal density is large compared to the longitudinal modal density, then the pattern of behaviour is reversed; i.e., the energy flow due to a flexural incident wave is minimum when the beams are at right angles and maximum when they are in parallel, while the energy flow due to a longitudinal incident wave is minimum when the beams are in parallel and maximum when they are at right angles. These results agree well with those presented by Horner and White for a wave model of two semi-infinite beams joined together.

\section{REFERENCES}

1. R. S. LANGLEY 1989 Journal of Sound and Vibration 135, 499-508. A general derivation of the Statistical Energy Analysis equations for coupled dynamic systems.

2. K. Shankar and A. J. Keane 1995 Journal of Sound and Vibration 185, 867-890. Energy flow predictions in a structure of rigidly joined beams using receptance theory.

3. J. M. CusChiER 1992 Journal of the Acoustical Society of America 91(5), Parametric analysis of the power on an L-shaped plate using a mobility power flow approach.

4. H. G. D. Goyder and R. G. White 1980 Journal of Sound and Vibration 68, 59-75. Vibrational power flow from machines into built up structures; part 1: Introduction and approximate analysis of beam and plate like foundations.

5. L. Cremer, M. Heckl and E. E. Ungar 1988 Structure Borne Sound. Berlin: Springer-Verlag; second edition.

6. J. L. Horner, R. G. White, R. Hooke and T. A. JeEves 1960 Journal of Sound and Vibration 147, 87-103. Direct search solution of numerical and statistical problems.

7. H. G. DAviEs 1972 Journal of the Acoustical Society of America 51, 387-392. Exact solutions for the response of some coupled multimodal systems.

8. H. G. DavIES 1972 Journal of the Acoustical Society of America 51, 393-401. Power flow between two coupled beams.

9. A. J. Keane 1992 Proceedings of the Royal Society of London A436, 537--568. Energy flows between arbitrary configurations of conservatively coupled multi-modal elastic subsystems.

10. L. Gaul 1983 Journal of Vibration, Acoustics, Stress, and Reliability in Design 105, 489-496. Wave transmission and energy dissipation at structural and machine joints.

11. M. Yoshimura and T. OKuShima 1977 Annals of the CIRP 25, 193-198. Measurement of dynamic rigidity and damping property for simplified joint models and simulation by computer.

12. F. J. FAHY and D. YAO 1987 Journal of Sound and Vibration 112, 1-11. Power flow between nonconservatively coupled oscillators.

13. J. C. Sun, N. Lalor and E. J. Richards 1987 Journal of Sound and Vibration 112, 321-330. Power flow and energy balance of nonconservatively coupled structures; 1: theory.

14. R. H. Lyon 1975 Statistical Energy Analysis of Dynamic Systems: Theory and Applications. Cambridge, MA: MIT Press.

15. R. C. N. LeUng and R. J. Pinnington 1990 Journal of Sound and Vibration 142, 31-46. Wave propagation through right-angled joints with compliance: flexural incident wave. 
16. R. C. N. Leung and R. J. Pinnington 1992 Journal of Sound and Vibration 153, 223-237. Wave propagation through right-angled joints with compliance: longitudinal incident wave.

17. T. E. RoOK and R. SingH 1995 Journal of the Acoustical Society of America 97, 2882-2891 Power flow through multidimensional compliant joints using mobility and modal approaches.

18. M. Beshara and A. J. KeANe 1996 Journal of Sound and Vibration 198, 95-122. Statistical energy analysis of multiple, non-conservatively coupled systems.

19. L. L. Howell and A. MidHa 1994 Journal of Mechanical Design 116, 280-290. A method for the design of compliant mechanisms with small-length flexural pivots.

20. M. Beshara, G. Y. Chohan, A. J. Keane and W. G. Price 1997 in Statistical Energy Analysis: an Overview with Applications in Structural Dynamics (A. J. Keane and W. G. Price, editors) Cambridge: Cambridge University Press. Statistical energy analysis of nonconservative systems.

21. J. L. HORNER and R. G. WHITE 1990 International Journal of Mechanical Sciences 32, 21 5-223. Prediction of vibrational power transmission through jointed beams. 